\title{
Initial stages of self-assembled InAs/InP(001) quantum wire formation
}

David Fuster ${ }^{\mathrm{a}, *}$, Benito Alén ${ }^{\mathrm{a}}$, Luisa González ${ }^{\mathrm{a}}$, Yolanda González ${ }^{\mathrm{a}}$, and Juan Martínez-Pastor ${ }^{\mathrm{b}}$

a Instituto de Microelectrónica de Madrid (CNM, CSIC), Isaac Newton 8, 28760, Tres Cantos, Madrid, Spain.

${ }^{\mathrm{b}}$ Instituto de Ciencia de los Materiales, Universidad de Valencia, P. O. Box 2085, 46071 Valencia, Spain.

\begin{abstract}
In this work we explore the first stages of quantum wires (QWRs) formation studying the evolution of the growth front for InAs coverage below the critical thickness, $\theta_{c}$, determined by reflection high energy electron diffraction. Our results show that at a certain InAs coverage $\theta(\operatorname{InAs})<\theta_{c}$, the elastic relaxation process starts by spontaneous formation of isolated quantum wires that evolves towards QWRs covering the whole surface with increasing InAs thickness. These results allow for a better understanding of the self-assembling process of QWRs in the InAs/InP system and for the first time enable the study of the novel properties of single self-assembled QWR.
\end{abstract}

PACS: 78.67.Lt; 68.65.La; 68.37.Ps;

Keywords: A1. Nucleation; A1. Nanostructures; A3. Molecular Beam Epitaxy; B2. Semiconducting indium phosphide;

\footnotetext{
* Corresponding author. E-mail address: davidf@imm.cnm.csic.es (D. Fuster)
} 


\section{Introduction}

The growth of heteroepitaxial systems with lattice mismatch larger than $2 \%$ has been extensively used for obtaining self-assembled nanostructures. There are a lot of theoretical and experimental works dedicated to study this self-assembling process [1-3], which results in more or less ordered distributions of quantum wires or quantum dots.

The self-assembled nanostructures based on the InAs/InP (001) heteroepitaxial system have a great technological interest as it is possible to tune the emission wavelength in a wide range $(1.2<\lambda<1.9 \mu \mathrm{m})$ at room temperature [4]. In this system, under molecular beam epitaxy (MBE) growth conditions, an array of [1-10] oriented quantum wires (QWRs) covering the whole surface is obtained when a certain InAs critical thickness, $\theta_{c}$, is grown. This critical thickness is detected by a 2D-3D sudden change in the reflection high energy electron diffraction (RHEED) pattern [5]. The spontaneous formation of QWR instead of quantum dots is due to the intrinsic strain asymmetry built-in at the InAs/InP interface under $\mathrm{V}$ element stabilized growth conditions [6]. Due to their shape, these structures allow for elastic relaxation along [110] remaining fully strained along [1-10] [6,7].

In this work, we explore the first stages of QWRs formation studying by atomic force microscopy (AFM) characterization the evolution of the growth front for certain InAs coverages below the $\theta_{c}$ determined by RHEED. Our results, supported by optical characterization of the similar buried nanostructures, show that at certain InAs coverage $\theta(\operatorname{InAs})<\theta_{c}$, spontaneous formation of isolated QWR are obtained. When the amount of InAs is increased, these isolated QWRs evolve towards groups of longer and narrower wires that progressively cover the surface until a full coverage is obtained at $\theta(\operatorname{InAs})$ $=\theta_{\mathrm{c}}$.

\section{Experimental}

The samples studied in this work were grown by solid source MBE and consist of different amount of InAs : 1.3, 1.5, 1.7, and 2.5 monolayers (ML) deposited on $\operatorname{InP}(001)$ substrates. Before InAs growth, the InP surface was exposed to $\mathrm{As}_{4}$ flux during $3 \mathrm{~s}$ at substrate temperature $\mathrm{T}_{\mathrm{S}}=480{ }^{\circ} \mathrm{C}$. This procedure allow us to control the InAs quantity ( 1 ML) formed by As/P exchange at the InAs/InP interface, which, besides the InAs thickness deposited, takes part in the QWRs self-assembled formation process. This means that the effective InAs thickness, $\theta$ (InAs), involved in the nanostructure formation process is $\theta(\operatorname{InAs}) \approx 2.3$, 2.5, 2.7 and 3.5 ML (samples A-D) for the different samples under study. InAs was deposited at a growth rate $\mathrm{r}_{\mathrm{g}}(\mathrm{InAs})=0.5 \mathrm{ML} / \mathrm{s}, \mathrm{T}_{\mathrm{S}}=480{ }^{\circ} \mathrm{C}$ and beam equivalent pressure BEP(As $\left.\mathrm{As}_{4}\right)$ $=4 \times 10^{-6}$ mbar. After InAs deposition, the surface was kept at $\mathrm{T}_{\mathrm{S}}=480{ }^{\circ} \mathrm{C}$ during 1.5 minutes under $\mathrm{As}_{4}$ flux. The surface topography of these samples were studied by atomic force microscopy (AFM)

Similar samples, but covered with a $20 \mathrm{~nm}$ thick InP cap layer grown by atomic layer MBE (ALMBE) at $\mathrm{T}_{\mathrm{S}}=380{ }^{\circ} \mathrm{C}$ were fabricated for photoluminescence (PL) characterization (samples $\mathrm{A}_{\mathrm{c}^{-}}-\mathrm{D}_{\mathrm{c}}$ ). We have used non-resonant excitation at $514.5 \mathrm{~nm}$ under low power conditions. The PL signal was dispersed by a $0.22 \mathrm{~m}$ focal length monochromator and synchronously detected with a liquid nitrogen cooled Ge detector. 


\section{Results and discussion}

Figure 1(a-d) shows $1 \mathrm{x} 1 \mu \mathrm{m}^{2}$ AFM images of the uncapped samples A-D respectively. The InAs surface of sample A [ $\theta(\operatorname{InAs})=2.3 \mathrm{ML}]$ shows elongated [1-10] islands formed at the edges of the $1 \mathrm{ML}$ high steps existing at the surface due to the unintentional miscut angle of the $\operatorname{InP}(001)$ wafer $\left(\sim 0.1^{\circ}\right.$ off). Similar structures were reported by exposing an InP surface to arsenic flux by Yang et al. [8].

The elongated dendrite-like islands shown on Fig. 1(a) are the precursors of the isolated asymmetric structures observed in the AFM image of sample B with $\theta(\operatorname{InAs})=2.5$ ML [Fig. 1(b)] These structures begin to form mainly at the islands edges, though a few of them have also been observed on the flat terraces. Due to the intrinsic built-in asymmetric strain at the InAs/InP interface [6], the critical accumulated stress is firstly achieved in [110]; this happens when the InAs island edge reaches a height between 2 and $3 \mathrm{ML}$ according to the measurements obtained for the isolated asymmetric structures shown in Fig. 1(b). This result agrees with that reported by Porte [3] where the 2D-3D transition occurs when the number of $M L$ at island edge $\left(\mathrm{N}_{\text {edge }}\right)$ reaches a critical value. However, along [1-10] the QWRs continue growing even passing across the $1 \mathrm{ML}$ step height of the 2D InAs terraces [see profile in Fig. 1(b)], because in this direction the critical accumulated stress for elastic relaxation has not already been reached at this InAs thickness.

A statistical study of the dimensions from more than 50 of these structures gives a mean height of 0.7 \pm 0.2 and width $=22 \pm 5 \mathrm{~nm}$. The average ratio length/width is around 8, but we can find longer structures [Fig. 1(b)] with a ratio of 16. Thus, even at the early stages of the self-assembling process, for $\theta(\operatorname{InAs})=2.5 \mathrm{ML}<\theta_{\mathrm{c}}$, elastic relaxation process leads to the formation of InAs nanostructures that can be considered as 1D systems (quantum wires). In this respect, an important result is the low QWRs areal density. For example, in Fig. 1(b) we find 14 QWR in an area of $1 \mathrm{x} 1 \mu \mathrm{m}^{2}$. Such small density will allow resolving the emission of a single QWR using high resolution optical techniques [9].

For the sample with $\theta(\operatorname{InAs})=2.7 \mathrm{ML}$ of InAs (sample C) we find coexisting isolated QWRs together with others that being longer and narrower, remain constant in height [Fig. 1(c)]. These QWRs are grouped in bundles of QWRs periodically separated. The increase in length and density of the QWRs observed in this sample indicates a significant increment in the amount of InAs involved, even if only 0.2 ML has been added to the InAs deposited in sample B. Driven by stress relaxation, the short and isolated QWRs act as sinks for preferential InAs growth not only for the incoming In and As atoms, but also by promoting mass transport by enhanced diffusion from 2D areas to the 3D structures $[4,10]$. When the amount of $\theta$ (InAs) is increased up to 3.5 ML (sample D) we observe chevrons in the RHEED pattern, corresponding to the conventional concept of critical thickness. In this situation, we obtain the typical array of QWRs covering completely the surface [Fig. 1(d)] with their typical geometrical parameters (1.1 $\pm 0.3 \mathrm{~nm}$ high, $12 \pm 3 \mathrm{~nm}$ wide) [11]. The observed dependence of the width of the QWR on the proximity of other QWR indicates that the interplay of the elastic interactions between close QWRs determine an equilibrium shape and separation between them.

As we will show below, the PL experiments from the capped samples (shown in Fig. 2) support the process of InAs nanostructures formation resulting from the AFM results. 
We can distinguish multiple peaks that can be associated to the emission from two different structures: quantum wells (QWs) and quantum wires. The PL spectrum of sample $\mathrm{A}_{\mathrm{c}}$ with $\theta(\mathrm{InAs})=2.3$ ML shows two main peaks [QW2 and QW3 in Fig. 2(a)]. Since QWR have been rarely found by AFM in this sample, we associate these two peaks to the emission from 2 and 3 ML high QWs in view of the surface morphology and previous studies reported in the literature for InAs/InP QWs [12]. Radiative recombination takes place in this case in the flat surface islands observed in the AFM image [Fig. 1(a)]. The sample $A_{c}$ already shows several weak PL peaks that could be associated to the emission of QWRs, but these bands are more clearly seen in the PL spectrum from sample $B_{c}$ [Fig. 2(b)]. With 2.5 MLs of InAs, in this sample the QWR emission gains in relevance compared with the emission from the 2 and 3 ML quantum wells (QW2 and QW3). The QWR emission is composed of three bands at 0.912, 0.863 and $0.830 \mathrm{eV}$, respectively. It is worth noting that their linewidths are noticeably larger $(>22 \mathrm{meV})$ than those of the QW bands (12 and $15 \mathrm{meV}$, respectively), as expected due to the larger inhomogeneous broadening expected for QWRs.

For sample $\mathrm{C}_{\mathrm{c}}$ [Fig. 1(c)], with a $\Delta \theta(\operatorname{InAs})=0.2 \mathrm{ML}$ respect to sample $\mathrm{B}_{\mathrm{c}}$, the QW2 and QW3 peaks are almost quenched in favor of the emission of the QWRs band. In this region, four bands are clearly resolved. Their peak energies agree well with QWRs height families of 8, 9, 10 and 11 MLs. [13,14] Comparing both samples, we observe that while the lowest energy peaks remain at their initial positions, the band at $0.912 \mathrm{eV}$ has been substituted by two new peaks, at $0.934 \mathrm{eV}$ and $0.897 \mathrm{eV}$, that now dominate the PL spectrum. The evolution just shown actually confirms that the InAs forming these QWRs comes not only from the incoming As and In fluxes, but also at the expense of mass transport from the flat areas, as discussed previously from the AFM results. It also suggests that during the inital stages of the self-assembling proccess the formation of large (thick) QWRs occurs first than the small ones.

This result agrees with a larger QWRs density as observed in the AFM image from the corresponding uncapped sample [Fig. 1(b)]. In the QWRs emission band, up to three peaks can be resolved, which are associated to three different height families: 9, 10 and 11 ML [13,14]. Notice that these heights do not coincide with those measured by AFM. In fact, AFM QWR heights in uncapped samples are systematically smaller than the obtained by TEM or expected from the PL results in capped samples [15]. We think that overgrowth of the QWRs takes place during the capping process particularly at the low substrate temperature used $\left(\mathrm{T}_{\mathrm{S}}=380^{\circ} \mathrm{C}\right)[16]$.

Finally, in Fig. 2(d), the PL spectrum of sample $\mathrm{D}_{\mathrm{c}}$ shows the typical PL band formed by the multiple gaussian peaks emitting from different QWR families with 1 ML fluctuations in height $[4,11,13,15]$. Notice that no emission from quantum wells is observed, in agreement with the absence of flat areas in a surface completely covered by QWRs [see Fig. 1(d)].

\section{Summary}

When a certain InAs thickness, $\theta c$, is grown on $\operatorname{InP}(001)$, an array of [1-10] oriented quantum wires covering the whole surface is obtained. This $\theta c$ corresponds to a sudden change in the RHEED pattern from $2 \mathrm{D}$ to $3 \mathrm{D}$. 
Using AFM and PL techniques, in this work we have explored the process of QWRs formation for InAs coverage below the critical thickness $\theta_{c}$, i.e., the intermediate states between a starting flat 2D surface and a surface fully covered by QWRs.

We have identified different stages that take place with increasing $\theta(\operatorname{InAs})$. First stage is the formation of islands elongated along [1-10] at the step edges of the flat terraces present in the surface. Next stages upon further InAs deposition are characterized by the formation of different nanostructures with a clear $1 \mathrm{D}$ aspect ratio. When the step height at the edge of the elongated islands reaches 2 or $3 \mathrm{ML}$, stress relaxation takes place along [110] direction. At this stage, QWR-like isolated nanostructures appear. Increasing $\theta(\operatorname{InAs})$, single QWR transform into bundles of narrower, longer but with constant height QWR that progressively cover the surface. For $\theta(\operatorname{InAs})=3.5 \mathrm{ML}$ a clear $2 \mathrm{D}-3 \mathrm{D}$ change is observed in the RHEED pattern and the whole surface is covered by QWR.

The main result of this work is the formation of self-assembled isolated QWR as an elastic relaxation step previous to the well established process of nanostructures formation, conventionally related to a critical thickness determined by an abrupt change in the RHEED pattern.

In conclusion, these results allow for a better understanding of the self-assembling process of QWRs in the InAs/InP system and for the first time will enable the study of the novel properties of selfassembled individual quantum wires.

\section{Acknowledgements}

The authors gratefully acknowledge financial support by the Spanish MEC and CAM through projects No. TEC-2005-05781-C03-01, NAN2004-09109-C04-01 and S- 505/ESP/000200, and by the European Commission through SANDIE Network of Excellence (No. NMP4-CT-2004-500101). 


\section{References}

[1] D. J. Bottomley, Appl. Phys. Lett. 72 (1997) 783.

[2] V. A. Shchukin and D. Bimberg, Rev. Mod. Phys. 71 (1999) 1125.

[3] L. Porte, J. Crystal Growth 273 (2004) 136.

[4] D. Fuster, L. González, Y. González, M. U. González, and J. Martínez-Pastor, J. Appl. Phys. 98 (2005) 033502

[5] H. R. Gutiérrez, M. A. Cotta, and M. M. G. de Carvalho, Appl. Phys. Lett. 79 (2001) 3854.

[6] J. M. García, L. González, M. U. González, J. P. Silveira, Y. González, and F. Briones, J. Crystal Growth 227 (2001) 975 .

[7] A. Mazuelas, L. González , J.M. García , Y. González , T. Schuelli , C. Priester, and H.T.Metzger, Phys. Rev. B 73 (2006) 045312.

[8] H. Yang, P. Ballet, and G. J. Salamo, J. Appl. Phys. 89 (2001) 7871.

[9] B. Alén, D. Fuster, Y. González, L. González, J. Martínez-Pastor, M. U. González, and J. M. García, Preprint available at cond-mat/0606134.

[10]D. Fuster, M. U. González, L. González, Y. González, T. Ben, A. Ponce, and S. I. Molina, Appl. Phys. Lett. 84 (2004) 4723.

[11]L. González, J. M. García, R. García, F. Briones, J. Martínez-Pastor, C. Ballesteros, Appl. Phys. Lett. 76 (2000) 1104.

[12] M. Hopkinson, J. P. R. David, P. A. Claxton, and P. Kightey, Appl. Phys. Lett. 60 (1991) 841.

[13] B. Alén, J. Martínez-Pastor, A. García-Cristóbal, L. González, and J. M. García, Appl. Phys. Lett. 78 (2001) 4025.

[14] J. Maes, M. Hayne, Y. Sidor, B. Partoens, F. M. Peeters, Y. González, L. González, D. Fuster, J. M. García, and V. V. Moshchalkov, Phys. Rev. B 70 (2004) 155311.

[15]D. Fuster, M. U. González, L. González, Y. González, T. Ben, A. Ponce, S. I. Molina, and J. Martínez-Pastor, Appl. Phys. Lett. 85 (2004) 1424.

[16] W. Seifert, D. Hessman, X. Liu, and L. Samuelson, J. Appl. Phys. 75 (1993) 1501. 


\section{Figure captions}

Fig. 1. 1x1 $\mu^{2}$ AFM images (a-d) from samples A-D with 2.3, 2.5, 2.7 and 3.5 monolayers of InAs grown on $\operatorname{InP}(001)$ respectively. Profiles along the direction of the dashed lines drawn on (a) and (b) are also shown. The elongated islands at the step edges (indicated by a white dotted line) (a) and the quantum wires (b-d) are always aligned along [1-10].

Fig. 2. Photoluminescence at $\mathrm{T}=12 \mathrm{~K}$ from samples $\mathrm{A}_{\mathrm{c}}-\mathrm{D}_{\mathrm{c}}$, with 2.3, 2.5, 2.7 and 3.5 monolayers of $\operatorname{In} \mathrm{As}$ respectively, covered by a $20 \mathrm{~nm}$ thick InP layer (see Fig. 1 for topographic images from the corresponding uncapped samples). Notice the progressive loss of PL intensity from the quantum wells (QWs) in favor of the PL emission from the quantum wires (QWRs) with increasing $\theta$ (InAs). 
ThA3-1

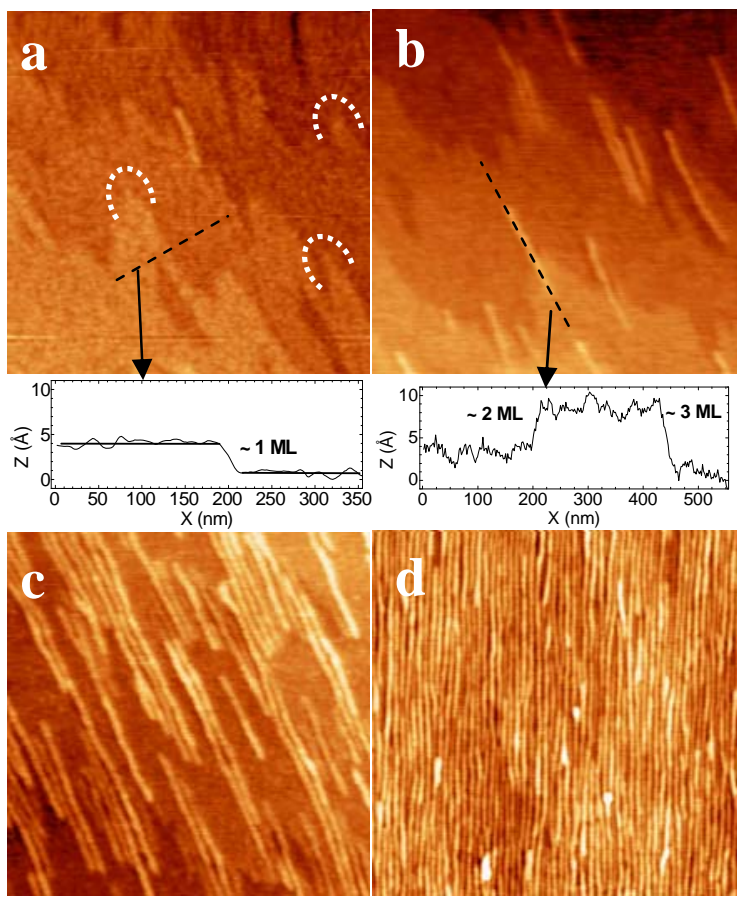

Figure 1. 


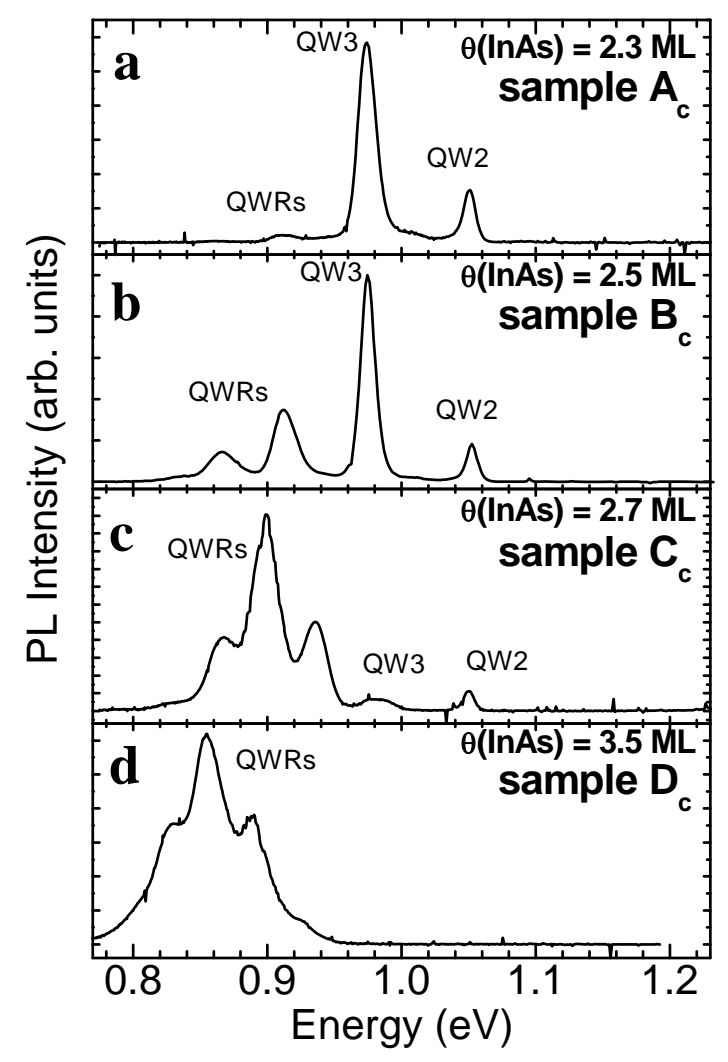

Figure 2. 